\title{
Changing Trends in Lower Gastrointestinal Hemorrhage: A Rise in Frequency of Ischemic Colitis
}

\author{
John Vizuete, John Alvarez, Charles Randall \\ Department of Internal Medicine, UT Health Science Center San Antonio, San Antonio, USA \\ Email: jialvarez915@gmail.com
}

Received 21 March 2014; revised 27 April 2014; accepted 5 May 2014

Copyright (C) 2014 by authors and Scientific Research Publishing Inc.

This work is licensed under the Creative Commons Attribution International License (CC BY). http://creativecommons.org/licenses/by/4.0/

(c) (i) Open Access

\section{Abstract}

The range and distribution of sources of lower gastrointestinal bleeding (LGIB) seem to be evolving over time. Ischemic colitis (IC) has long been recognized as a common cause of LGIB. Due to a variety of contributing factors, we suspect that IC may be implicated in an increasing proportion of inpatient cases of lower GI bleeding compared to previously published rates. We examined the medical records of 464 patients admitted to the Methodist Hospital from 2005 to 2013 . Patients with LGIB admitted to the hospital with a diagnostic colonoscopy were eligible for the study. Demographics and diagnoses were grouped and categorized for ease of comparison and compatibility with prior studies. Statistical analysis was used to summarize disease distribution and calculate probability outcomes based on pooled mean values obtained from 6 previously published epidemiological studies of LGIB. Anorectal bleeding including hemorrhoids, fissures, and stercoral ulcers was the most frequent diagnosis overall $(20 \%)$ followed by ischemic colitis (16\%) and diverticulosis (14\%). There were a significantly higher proportion of IC cases observed in our population compared to the expected proportion of cases $(p<0.01,95 \%)$. When stratified by sex, IC was the leading overall cause in females.

\section{Keywords}

Lower Gastrointestinal Bleeding, Ischemic Colitis, Hematochezia

\section{Introduction}

Gastrointestinal bleeding (GIB) is a common clinical scenario encountered by general practitioners and specialists alike. GIB is divided into upper (UGIB) and lower (LGIB) sources based on the origin of bleeding in rela- 
tionship to the ligament of treitz. LGIB accounts for about 1\% of all hospital admissions [1]. This retrospective review will focus on etiologies of LGIB in the inpatient setting.

The epidemiology of lower gastrointestinal bleeding (LGIB) seems to be evolving over time. Historically, colonic diverticular bleeding has been considered the most common source, with reported incidence ranging from $20 \%-50 \%$ [2]. Angiodysplasia, anorectal sources, neoplasia (whether benign or malignant), and acute inflammatory processes (infection, Crohn's disease, ulcerative colitis) are other known causes. In recent years, ischemic colitis has been recognized as an increasingly prevalent disease process.

Ischemic colitis was first described as a cause of LGIB in 1963 by Boley et al. [3]. Reported rates have varied from $6 \%-19 \%$ and older studies tend to report lower rates [4]-[9]. The mechanism of ischemic colitis is distinct from acute or chronic mesenteric ischemia, the result of decreased arterial perfusion of the gut. Instead, increased pressure on the gut wall, often due to constipation and slow fecal transit time, results in mucosal ischemia and sloughing, clinically manifested as hematochezia. The diagnosis is confirmed on histology by mucosal and/or submucosal hemorrhage, frequently with a mild lymphocytic infiltration [10].

Due to a variety of contributing factors, we suspect that ischemic colitis may be occurring more frequently in the inpatient setting compared to previously published data for LGIB.

\section{Methods}

We examined electronic medical records of hospitalized patients seen by physicians in our group from January 2005 to October 2013. Patients with ICD-9 diagnosis codes of 569.3 (rectal hemorrhage) or 578.9 (hematochezia) who underwent colonoscopy for evaluation were included in the study. We did not distinguish whether patients were admitted primarily for bleeding or if they developed bleeding while in the hospital for an alternative reason. No measurement of bleeding severity was applied. Some gastroenterologists in our group specialize in inflammatory bowel disease (IBD); therefore to avoid sampling bias, patients with a known history of IBD were excluded from the study.

Data for patient age, sex, and final diagnosis as documented on the operation report, pathology report (when biopsies were taken), or discharge summary were collected. A clinical diagnosis was made in the case where a biopsy proved to be equivocal or when no biopsy was taken. Diagnoses were grouped into 10 categories for ease of comparison with prior studies. The categories were formulated as follows: anorectal causes (which included internal and external hemorrhoids, anal fissures and stercoral ulcers), ischemic colitis, diverticulosis, neoplasia (benign and malignant etiologies), upper gatrointestinal bleeding (UGIB), other causes (which included graft versus host disease, radiation proctitis, post biopsy bleed), arteriovenous malformation (AVMs), IBD, non-specific colitis (includes infectious etiologies or biopsy report of "non-specific colitis"). Frequencies of each diagnostic category were recorded. If there was no definitive diagnosis (i.e., multiple potential causes) or if the diagnosis was unclear (i.e., no bleeding source found), then these patients were placed in the unclear category. To analyze whether there was a significant difference in the proportion of IC cases observed in this study from what has been documented in previous literature, we compared pooled data from six published epidemiologic studies of LGIB to our patients using a two sample z-test [2] [4]-[8]. Data were analyzed with Stata V.11 and Microsoft Excel 2010 Analysis Toolpak.

\section{Results}

464 patients met criteria for review. 208 (45\%) were male and 256 (55\%) were female (Table 1). The average age was $64.8 \pm 17.9$ years. Elderly females ( $>65$ years of age) represented a third of the patient population. A definitive diagnosis was made in $79 \%$ of patients. In the remaining $21 \%$, the origin of bleeding remained unclear-either no source was identified or multiple potential but inactive sources were noted (e.g., hemorrhoids and diverticulae). In all comers, an anorectal source of bleeding including hemorrhoids, fissures, or stercoral ulcers was the leading diagnostic category at $20 \%(n=96)$. Ischemic colitis $(16 \%)$ followed by diverticular bleeding (14\%) were the second and third most frequent etiologies, respectively (Table 2).

When stratified by age, diverticulosis (19\%), followed closely by ischemic colitis (18\%) and anorectal causes (16\%) were most common in patients $>65$ years (Table 3 ). When stratified by gender, ischemic colitis was the most frequently diagnosed etiology in women (24\%). In men of all ages, an anorectal source was the most common diagnosis (20\%), though this category was surpassed by diverticular bleeding in men $>65$ years of age (Table 4). There was a significant difference seen between the proportion of ischemic colitis cases observed in 
Table 1. Demographics.

\begin{tabular}{cccc}
\hline Sex & N & (\%) \\
\hline Female & 256 & 55 \\
Male & 208 & 45 \\
Total & 464 & 100 \\
\hline Age & & \\
\hline$<65$ & 202 & 43 \\
$>65$ & 262 & 57 \\
Total & 464 & 100 \\
\hline
\end{tabular}

Table 2. Percentage of patients represented by diagnosis category.

\begin{tabular}{|c|c|c|}
\hline Diagnosis & $\mathbf{N}$ & (\%) \\
\hline Unclear source $^{\mathrm{a}}$ & 96 & 21 \\
\hline Anorectal $^{\mathrm{b}}$ & 91 & 20 \\
\hline Ischemic colitis & 75 & 16 \\
\hline Diverticulosis $^{c}$ & 63 & 14 \\
\hline Neoplasia $^{\mathrm{d}}$ & 31 & 7 \\
\hline Other $^{e}$ & 29 & 6 \\
\hline UGIB & 25 & 5 \\
\hline AVMs & 21 & 5 \\
\hline IBD & 19 & 4 \\
\hline Non-specific colitis ${ }^{f}$ & 14 & 3 \\
\hline Total & 464 & 100 \\
\hline
\end{tabular}

${ }^{\mathrm{a}}$ When the source of bleeding was not found (e.g., normal colonoscopy) or multiple potential sources existed simultaneously (diverticulosis and non-bleeding hemorrhoids). ${ }^{\mathrm{b}}$ Includes internal hemorrhoids, anal fissures, stercoral ulcers. ${ }^{\mathrm{C}}$ Patients with non-bleeding diverticulae were included in the "unclear" category. ${ }^{\mathrm{d}}$ Benign or malignant. ${ }^{\mathrm{e}}$ Includes radiation proctitis, GVHD, post-biopsy rebleeding. ${ }^{\mathrm{f}}$ Includes infectious etiologies or biopsy report of "non-specific" colitis.

\section{Table 3. Distribution by gender as well as age.}

\begin{tabular}{|c|c|c|c|c|}
\hline \multirow{2}{*}{ Diagnosis } & \multicolumn{4}{|c|}{ Frequency (\%) } \\
\hline & Female (\%) & Male (\%) & Age $<65(\%)$ & Age 65+ (\%) \\
\hline Ischemic colitis & $61(24)$ & $14(7)$ & $28(14)$ & $47(18)$ \\
\hline Anorectal & $50(20)$ & $41(20)$ & $49(24)$ & $42(16)$ \\
\hline Diverticulosis & $30(12)$ & $33(16)$ & $12(6)$ & $51(19)$ \\
\hline Neoplasia & $18(7)$ & $13(6)$ & $16(8)$ & $15(6)$ \\
\hline AVMs & $12(5)$ & $9(4)$ & $5(2)$ & $16(6)$ \\
\hline UGIB & $12(5)$ & $13(6)$ & $11(5)$ & $14(5)$ \\
\hline IBD & $12(5)$ & $7(3)$ & $16(8)$ & $3(1)$ \\
\hline Non-specific colitis & $7(3)$ & $7(3)$ & $9(4)$ & $5(2)$ \\
\hline Other & $10(4)$ & $19(9)$ & $15(7)$ & $14(5)$ \\
\hline Unclear source & $44(17)$ & $52(25)$ & $41(20)$ & $55(21)$ \\
\hline Total & $256(100)$ & $208(100)$ & $202(100)$ & $262(100)$ \\
\hline
\end{tabular}


our population compared to what has been shown in prior literature [(16\% vs. $11 \%, p<0.01,95 \%$ ) (Table 5)]. Notably, diverticular bleeding was definitively identified far less than in previous studies (14\% vs. $27 \%, p<$ $0.01,95 \%)$.

\section{Discussion}

Our population was similar in age to previously studied groups, but exhibited a slightly higher female predominance. With the exception of ischemic colitis and diverticular bleeding, the disease distribution in our population was similar to prior studies. Though the prevalence of ischemic colitis appears to be rising, anorectal bleeding continues to be the most common source in hospitalized patients. Many patients, over 1 in 5 in this series, will not have a clear source despite a complete evaluation, although there is a significant body of literature to support that early colonoscopy can increase diagnostic yield [4] [11]. This may be because that unlike UGIB, $75 \%$ - 80\% of LGIB will resolve spontaneously, and leave fewer diagnostic clues to be seen on colonoscopy [12]. Interestingly, ischemic colitis was the most commonly encountered diagnosis in all women. Women disproportionately represented the majority of ischemic colitis cases seen (81\%). Only $14 \%$ of our population was found to have a diverticular source of bleeding, previously reported to be the most common cause of LGIB. This discrepancy may be explained in part by our categorization method. In the past, patients with an unclear source but the presence of diverticulosis on exam may have been counted as a diverticular bleeds. In this series, such a presumption was not made, and an inflamed or actively bleeding/oozing lesion was required. Additionally, some cases of potential diverticular bleeds were classified under the "unclear" category, due to the presence of alternative bleeding sources such as concomitant internal hemorrhoids or AVMs.

This study is subject to multiple limitations. The first is an inherent selection bias by including patients from a single facility. Additionally, we were unable to control for confounders such as ethnicity and socioeconomic sta tus. Gender bias may also influence our conclusion as females made up a larger segment of the study population.

Table 4. Distribution broken down to include gender with age.

\begin{tabular}{ccccc}
\hline \multirow{2}{*}{ Diagnosis } & \multicolumn{4}{c}{ Frequency (\%) } \\
\cline { 2 - 5 } & Females Age $<\mathbf{6 5}(\%)$ & Females Age $>\mathbf{6 5}$ (\%) & Males Age < 65(\%) & Males Age 65+ (\%) \\
\hline Ischemic colitis & $23(22)$ & $38(25)$ & $5(5)$ & $9(8)$ \\
Anorectal & $24(23)$ & $26(17)$ & $25(26)$ & $16(15)$ \\
Diverticulosis & $4(4)$ & $26(17)$ & $8(8)$ & $7(23)$ \\
Neoplasia & $9(9)$ & $9(6)$ & $7(7)$ & $6(5)$ \\
AVMs & $2(2)$ & $10(7)$ & $3(3)$ & $8(7)$ \\
UGIB & $6(6)$ & $6(4)$ & $7(5)$ & $0(0)$ \\
IBD & $9(9)$ & $3(2)$ & $4(4)$ & $3(3)$ \\
Non-specific colitis & $5(5)$ & $2(1)$ & $10(10)$ & $9(8)$ \\
Other & $5(5)$ & $5(3)$ & $24(24)$ & $28(25)$ \\
Unclear source & $17(16)$ & $27(18)$ & $98(100)$ & $110(100)$ \\
\hline Total & $104(100)$ & $152(100)$ & & \\
\hline
\end{tabular}

Table 5. Proportion of IC cases observed in the present study compared to data from pooled studies ${ }^{\mathrm{a}}$.

\begin{tabular}{cccc} 
& Number of IC Cases & Sample Size & Proportion \\
\hline Present study & 75 & 464 & $16.1 \%$ \\
Pooled studies $^{\mathrm{b}}$ & 200 & 1711 & $11.6 \%$ \\
\hline Total & 275 & 2175 & \\
\hline
\end{tabular}

${ }^{\mathrm{a}} \mathrm{A}$ two sample $\mathrm{Z}$ test was utilized to compare the proportion of IC cases in the present study to the pooled data (significant at $P<0.01$ ). ${ }^{\mathrm{b}} \mathrm{Data}$ retrieved from 6 studies on LGIB (2, 4 - 8). 
Finally, other factors such as diagnostic criteria, medical comorbidities and degree of hemorrhage may dramatically impact the likelihood of certain diagnoses, and must be considered alongside epidemiologic data in individual cases.

Colonic ischemia is directly related to hypoperfusion of the intestinal vasculature. Acute ischemia may be divided into occlusive or non-occlusive causes [13]. Occlusive etiologies include intravascular mechanical obstruction (thrombotic or embolic) in the setting of acute mesenteric ischemia or classically as a complication of aortic surgical procedures. Non-occlusive causes include hypotensive shock, volume depletion, impaired left ventricular function, or vasculitis among others. Ischemic colitis as defined in this study is distinct from these entities in that it is a mucosal phenomenon and is precipitated by increased intraluminal pressure (i.e., from constipation) based on LaPlace's law in addition to some non-occlusive factors mentioned above [14] [15].

In the past, ischemic colitis has been described as a disease of the elderly [16]. In both the young and elderly, ischemic colitis was the second most common diagnosis. These data suggest not only an increasing prevalence in all comers as might be expected with an aging population, but specifically in the segment of population under 65 . We suspect that this trend may be related to the effects of dehydration and constipation in the hospital. These data collectively demonstrate an increasing prevalence of ischemic colitis as a cause of LGIB.

\section{References}

[1] Vernava, A.M., Longo, W.E., Virgo, K.S., et al. (1996) A Nationwide Study of the Incidence and Etiology of Lower Gastrointestinal Bleeding. Surgical Research Communications, 18, 113-120.

[2] Strate, L.L. (2005) Lower GI Bleeding: Epidemiology and Diagnosis. Gastroenterology Clinics of North America, 34, 643-664. http://dx.doi.org/10.1016/j.gtc.2005.08.007

[3] Boley, S.J., Schwartz, S., Lash, J. and Sternhill, V. (1963) Reversible Vascular Occlusion of the Colon. Surgery, Gynecology \& Obstetrics, 116, 53-60.

[4] Strate, L.L. and Syngal, S. (2003) Timing of Colonoscopy: Impact on Length of Hospital Stay in Patients with Acute Lower Intestinal Bleeding. The American Journal of Gastroenterology, 98, 317-322.

[5] Newman, J.R. and Cooper, M.A. (2002) Lower Gastrointestinal Bleeding and Ischemic Colitis. Canadian Journal of Gastroenterology, 16, 597-600.

[6] Schmulewitz, N., Fisher, D.A. and Rockey, D.C. (2003) Early Colonoscopy for Acute Lower GI Bleeding Predicts Shorter Hospital Stay: A Retrospective Study of Experience in a Single Center. Gastrointestinal Endoscopy, 58, 841-846. http://dx.doi.org/10.1016/S0016-5107(03)02304-6

[7] Ohyama, T., Sakurai, Y., Ito, M., et al. (2000) Analysis of Urgent Colonoscopy for Lower Gastrointestinal Tract Bleeding. Digestion, 61, 189-192. http://dx.doi.org/10.1159/000007756

[8] Longstreth, G.F. (1997) Epidemiology and Outcome of Patients Hospitalized with Acute Lower Gastrointestinal Hemorrhage: A Population-Based Study. The American Journal of Gastroenterology, 92, 419-424.

[9] Tada, M., Shimizu, S. and Kawai, K. (1991) Emergency Colonoscopy for the Diagnosis of Lower Intestinal Bleeding. Gastroenterologia Japonica, 26, 121-124.

[10] Longo, W.E., Ballantyne, G.H. and Gusberg, R.J. (1992) Ischemic Colitis: Patterns and Prognosis. Diseases of the Colon \& Rectum, 35, 726-730. http://dx.doi.org/10.1007/BF02050319

[11] Habu, Y., Tahashi, K., Kiyota, K., et al. (1996) Reevaluation of Clinical Features of Ischemic Colitis: Analysis of 68 Consecutive Cases Diagnosed by Early Colonoscopy. Scandinavian Journal of Gastroenterology, 31, 881-886. http://dx.doi.org/10.3109/00365529609051996

[12] Billingham, R.P. (1997) The Conundrum of Lower Gastrointestinal Bleeding. Surgical Clinics of North America, 77, 214-252. http://dx.doi.org/10.1016/S0039-6109(05)70542-9

[13] Boley, S.J., Agrawal, G.P., Warren, A.R., et al. (1969) Pathophysiologic Effects of Bowel Distension on Intestinal Blood Flow. The American Journal of Surgery, 117, 228-234. http://dx.doi.org/10.1016/0002-9610(69)90308-0

[14] Green, B.T. and Tendler, D.A. (2005) Ischemic Colitis: A Clinical Review. Southern Medical Journal, 98, $217-222$. http://dx.doi.org/10.1097/01.SMJ.0000145399.35851.10

[15] Sreenarasimhaiah, J. (2005) Diagnosis and Management of Ischemic Colitis. Current Gastroenterology Reports, 7, 421-426. http://dx.doi.org/10.1007/s11894-005-0013-1

[16] Vernava, A., Moore, B., Longo, W., et al. (1997) Lower Gastrointestinal Bleeding. Diseases of the Colon \& Rectum, 40, 846-858. http://dx.doi.org/10.1007/BF02055445 